\title{
Thinking About Binary Compatibility and CentOS Stream
}

Pat Riehecky

CentOS Dojo May 2021

FERMILAB-SLIDES-21-020-SCD

14 May 2021 


\section{Disclaimers}

Pat Riehecky is not speaking as official spokesperson for:

- Fermi Research Alliance

- Fermi National Accelerator Laboratory

- US Department of Energy

Neither the United States nor the United States Department of Energy, nor any of their employees, makes any warranty, express or implied, or assumes any legal liability or responsibility for the accuracy, completeness, or usefulness of any data, apparatus, product, or process disclosed, or represents that its use would not infringe privately owned rights.

This is not an endorsement of any technology, software, service, or organization.

This presentation is an act of research, rather than a prescription of future practices. 


\section{The Road Ahead in this Presentation}

Nothing is more boring than a presentation where someone reads the slides to you...

Except perhaps when you already know most of what is on each and every slide.

Alas, I couldn't think of a better way to ensure a uniform understanding of the topics ahead, simplify translation for non-english speakers, and provide a way to use the slide deck as quick reference when people are under time pressure.

For the Ops people, this presentation may help you put the 'Dev' in 'DevOps'

For the Dev people, this presentation may help you put the 'Ops' in 'Dev Ops'

I've never worked at a place where folks complained about how thorough the communication is between Dev and Ops. Good communication is hard, just like good coordination. 


\section{Will my RHEL application run on CentOS Stream?}

In practice, the answer to this is a function of the RHEL ABI/API guarantee.

\section{https://access.redhat.com/articles/rhel8-abi-compatibility}

When using items on the RHEL approved list: if it runs on RHEL it should run on CentOS Stream without any modifications. Any incompatibilities there are bugs that need to be addressed. The "stable" ABI/API is about consistency or dependability.

This is a presentation about User Space.

Note: the RHEL Kernel is a whole separate discussion with a whole separate document outlining its ABI/API guarantees, a nice presentation yesterday also a blog post.

https://developers.redhat.com/blog/2018/03/28/analyzing-binary-interface-changes-linux-kernel/ 


\section{The RHEL8 ABI/API guarantee}

There are four levels outlined in the document:

Level 1: These are dependable within the lifetime of a major release and they are also dependable across the next two major releases (glibc, libxml2, etc)

Level 2: These are scoped within the lifetime of a single major release, but consistent within it (this is the default)

Level 3: Each component will specify a lifetime during which the ABI/API will be supported (AppStream/Modularity)

Level 4: No compatibility is guaranteed (gnome-desktop, libcgroup, libdrm, etc)

For the definitive rules and list of what binaries are in each group, you'll want to read the official documentation from Red Hat. 


\section{Will my CentOS Stream application run on RHEL?}

In practice, this too is a function of the RHEL ABI/API consistency guarantee, but the implementation is a bit different.

\section{https://access.redhat.com/articles/rhel8-abi-compatibility}

In order to answer this, we need to look at how binaries actually run on a Linux system and understand what that means in a RHEL context. 


\section{Anatomy of an ELF binary as a package}

For right now, we can treat an ELF binary as a type of runtime package. This should help us stay focused. After all, a binary RPM generally contains a collection of ELF binaries. We will be ignoring other binary types and ignoring ELF support/behavior on non Linux platforms.

The bits we care about at this time are the binary payload and the library linkage/symbols.

The binary payload contains the instructions to execute. If the CPU doesn't have the requested instructions, the binary will SEGFAULT once it hits an unknown instruction. RPM is not looking for these types of incompatibilities - I think that is a good thing. Microarchitectures (like x86_64v2) are interesting, but out of scope today. Generally speaking, the ' $x 86 \_64$ ' in the package arch is considered sufficient to confine it to CPUs it can use. For this presentation that is good enough for what we plan to explore.

Library linkages and symbol exports are a bit more complex to summarize and will consume the bulk of this presentation. 


\section{Anatomy of an ELF binary linkage}

There are a number of ways to review the dynamic libraries a binary uses and what various libraries provide.

- $l d d(-v)$

- objdump (-p/ -T)

- readelf $(-r /-s)$

NOTE: the ldd command will potentially execute elements of the object to achieve symbol linkage and is thus less safe on untrusted binaries than the alternatives. The manpages on ldd have more information on this. It is fascinating! You should read it!

You can also use abipkgdiff (from libabigail) to look at changes between two RPMs or binary objects. 


\section{Anatomy of an ELF linkage: an example with $x z$}

$\mathrm{xz}$ is a Compatibility Level Two package.

https://access.redhat.com/articles/rhel8-abi-compatibility

"Compatibility level 2

APIs and ABIs are stable within the lifetime of a single major release. Compatibility level 2 application interfaces will not change from minor release to minor release and can be relied upon by the application to be stable for the duration of the major release. Compatibility level 2 is the default for packages in Red Hat Enterprise Linux 8. Packages not identified as having another compatibility level may be considered compatibility level $2 . "$ 


\section{Anatomy of an ELF linkage: an example with $x z$}

As part of its inventory/dependency system, RPM utilizes objdump to review the linkage of each binary.

\$ objdump -p /usr/bin/xz \# from EL8

/usr/bin/xz: file format elf64-x86-64

[snip]

Version References:

required from libpthread.so.0:

0x09691a75 0x00 05 GLIBC_2.2.5

required from libc.so.6:

0x0d696917 0x00 07 GLIBC_2.7

0x06969197 0x00 06 GLIBC_2.17

0x09691974 0x00 04 GLIBC_2.3.4

0x09691a75 0x00 03 GLIBC_2.2.5

0x096a2561 0x00 09 GLIBC_2.32

required from liblzma.so.5:

0x05e02812 0x00 08 XZ_5.2

0x05e02810 0x00 02 XZ_5.0
The examples in this presentation will often switch back and forth between objdump and readelf style reporting.

I find a detailed investigation into the symbols is easier with readelf. Most system tools use objdump. You should use a tool you like and make sure to understand its limitations/risks. 


\section{Anatomy of an ELF linkage: an example with $x z$}

With readelf we can easily see exactly which foreign functions (library calls) are listed and specific information about them.

\$ readelf -r --wide /usr/bin/xz | awk '\{print \$5\}' | sort -u | grep XZ_ [snip]

lzma_stream_encoder_mt_memusage@xz_5.2

lzma_stream_flags_compare@Xz_5.0

lzma_stream_footer_decode@Xz_5.0

lzma_stream_header_decode@Xz_5.0

lzma_version_number@xz_5.0

lzma_version_string@Xz_5.0 


\section{Anatomy of an ELF linkage: an example with $x z$}

Putting together what we've uncovered about the xz binary on my test EL8 system:

- liblzma.so. 5 is used to resolve a number of symbols

- Some of those symbols are marked XZ_5.0 and some as XZ_5.2

- lzma_stream_encoder@Xz_5.0

- lzma_stream_encoder_mt@xz_5.2

Thankfully, liblzma follows the standard convention where the . 5 in . so. 5 corresponds to library version 5 .

Any system that provides the required $A B I$ symbols can run this binary.

Reminder: liblzma is a "Compatibility Level 2" library in RHEL 8. 


\section{Compatibility Level 2 in practice with liblzma}

EL7.0 xz on an EL8.0 host:

$\$ . / x z$

./xz: /lib64/liblzma.so.5: version 'Xz_5.1.2alpha' not found

Specifically: lzma_stream_encoder_mt@XZ_5.1.2alpha EL8.0 $x z$ on an EL7.0 host:

$\$ . / x z$

./xz: /lib64/libc.so.6: version 'GLIBC_2.32' not found

./xz: /lib64/liblzma.so.5: version 'Xz_5.2' not found

Specifically: 1zma_stream_encoder_mtexz_5.2

There is nothing necessarily unexpected here. Between major releases the liblzma symbols are permitted to change. Furthermore, the addition of new symbols to glibc in EL8 is expected and generally a good thing. These are new features! 


\section{A short detour into Level 1 (backwards compatible)}

glibc and libpthread are Level 1 libraries, so it is worth a brief detour to see what happened.

Opening up the $x z$ binary linkage :

In EL8 we see the addition of pthread_sigmask@GLIBC_2.32

In EL7 the binary has pthread_sigmask@GLIBC_2.2.5 instead

A look at the glibc changelog for libpthread shows:

* On Linux, the functions pthread_attr_setsigmask_np and

pthread_attr_getsigmask_np have been added. They allow applications

to specify the signal mask of a thread created with pthread_create.

Which in turn cascades down to pthread_sigmask causing the symbol addition.

The older symbol remains in the EL8 glibc (via libpthread), thus Level 1 rules are satisfied. 


\section{Compatibility Level 2 in practice with liblzma}

Looking at lzma_stream_encoder_mt

In this instance the solution is fairly trivial. As I understand the liblzma changelog, the symbol introduced in $x z-5.1$.2alpha was stabilized in $x z-5.2$ and saw no changes to the arguments or return structure.

In the end, the $\mathrm{ABI}$ of a binary is basically: the arguments, the return structure, the symbol name, and the namespace. In this instance three are the same - the namespace is what is different.

The code using this symbol name can be recompiled and relinked as is and should work exactly as expected.

But this isn't a conversation about porting software from EL7 to EL8.

This is a conversation about $A B I$ consistency. To keep matters simple, lets stick with liblzma and look at EL7 where we have a lot of history to examine. 


\section{Compatibility Level 2 in practice with liblzma on EL7}

In 2016 liblzma was rebased from 5.1.2-12alpha to version 5.2.2 in EL7.

The upstream source code has the symbols tagged as XZ_5.2. However, the EL7 source is patching the code to instead keep those symbols (lzma_stream_encoder_mt and lzma_stream_encoder_mt_memusage) marked with the XZ_5.1.2alpha namespace.

This rebase also adds some new symbols which it is tagging as a part of the XZ_5.2.2 namespace (which matches the version of liblzma where they were introduced into EL7). 


\section{Compatibility Level 2 in practice with liblzma on EL7}

xz-5.2.2-compat-libs.patch

this patch is a concise (and near perfect) example of Level 2 compatibility (author Pavel Raiskup?)

liblzma/api/lzma/container.h in 5.1.2alpha lists the 1zma_stream_encoder_mt and lzma_stream_encoder_mt_memusage as a part of their 'UNSTABLE' symbol namespace.

What does that mean? For liblzma upstream, These symbols are part of the 5.2 namespace. For EL7, they have to stay in 5.1.2alpha for Level 2 compatibility to be honored.

This slide is the centerpiece of this presentation
We provided two 5.1.2alpha symbols (lzma stream encoder mt and

lzma stream encoder mt memusage) before we updated to $x z-5,2,2$ - 1 in RHEL7.3.

Those symbols did not change ABI in 5.2 .2 so it should be safe to provide (except for 5.0 and 5.2 symbols) also the two 5.1.2alpha symbols and use 5.1.2alpha symbol version as parent for 5.2

For better reasoning look at container.h in 5.1.2alpha -. those two symbols were for testing purposes only, and thus not considered to be API/ABI.

diff --git a/src/liblzma/liblzma.map b/src/liblzma/liblzma.map

index f53a4ea..9c3002a 100644

a/src/liblzma/liblzma.map

$+++b / s r c / l i b l z m a / l i b l z m a, m a p$

@@ $-95,7+95,13$ @@ global:

\}

$-X Z 5.2\{$

$+X Z$ 5.1.2alpha \{

+global:

$+\quad$ lzma stream encoder mt;

lzma stream encoder mt memusage;

$+\}$ XZ_5.0

$+X Z$ 5.2.2

global:

lzma_block_uncomp_encode ;

lzma cputhreads;

(a) $-105,4+111,4$ @a global:

local:

- $X Z$ X 5.0;

+\} XZ_5.1.2alpha 


\section{Compatibility Level 2 in practice with liblzma on EL7}

The RPM dependencies for the $x z$ rpm are similarly interesting.

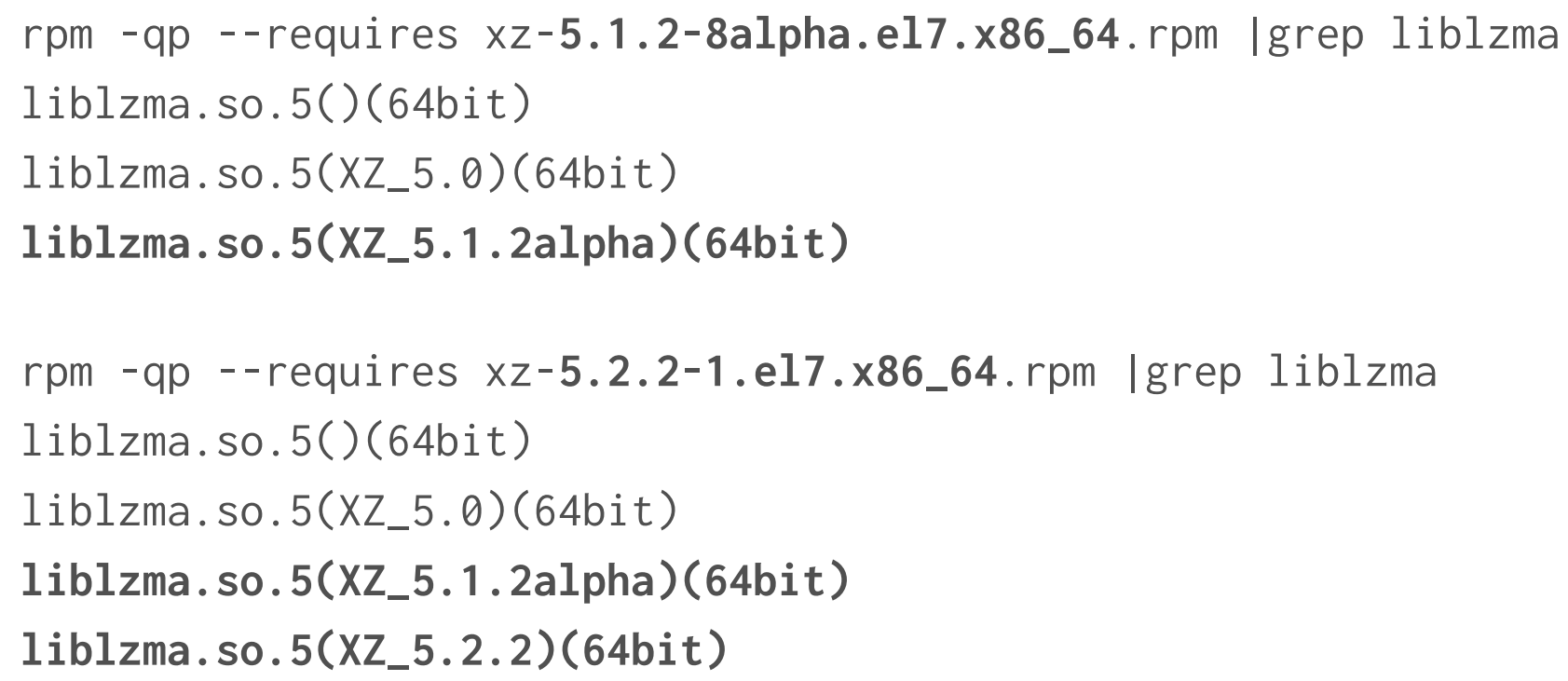

The new RPM correctly determines it needs the new symbols without the need to set an explicit version of liblzma under Require. 


\section{Compatibility Level 2 with liblzma in conclusion}

We've seen the source get rebased and have symbols added. The liblzma maintainer was required to honor the previous symbol names/namespaces and patched the old names back in.

We've seen that RPM correctly identifies the new symbols when used by new binaries.

We've also seen new binaries get the stable symbol names when built against the new libraries.

And we've seen that Level 2 libraries can in fact break between major releases.

The ABI/API guarantee is part of RHEL, and it worked as expected. 


\section{The ABI/API guarantee in CentOS Stream}

I think of CentOS Stream as the continuous delivery repository for RHEL. RHEL updates are published in bundles/batches/point releases on scheduled intervals. Stream is published now.

The packages in CentOS Stream are headed into RHEL. To my mind this means they must be suitable for running on RHEL.

Packages in CentOS Stream that violate the RHEL ABI/API guarantee are not suitable for running on RHEL.

Therefore, breakage of the RHEL ABI/API shouldn't happen in Stream.

If a CentOS Stream package violates the RHEL ABI/API guarantee the maintainer must fix it. This is not an optional step or something to be considered as an afterthought. If it doesn't get fixed, it changes in RHEL and if it changes in RHEL it violates RHEL contracts.

Packages in CentOS Stream that break the RHEL ABI/API are serious bugs. 


\section{The ABI/API guarantee in CentOS Stream: New symbols}

Maintenance of the existing ABI/API guarantees is well understood.

New symbols/features/ABI/API/etc are where things get complex.

Symbols not on the RHEL guarantee list are not guaranteed, but this doesn't mean they will change before going into RHEL - just that they can change. But how?

Changes to the arguments or return types don't feel like something you'd change from upstream. Similarly, the names of the symbols are selected by the upstream project. The namespace seems to be our only option for change here.

Use of new symbols presents a risk, but there are several mitigation strategies.

For developers they all boil down to "know what you are using".

For operations it is a question of how to provide extra libraries in a clean manner. 


\section{Mitigation: Developers}

What should developers do and think about? 


\section{Mitigation: Developers}

Step 1 is know what you are using.

This is already true for RHEL8 AppStream/Modularity.

If your application links against MariaDB/PostgreSQL you already must keep track of your version usage.

Similarly with gcc, $11 \mathrm{vm}$, python, perl, rust, swig, and more and more.....

Big questions:

Are the library features you selected "new" (ie not yet in RHEL)?

If they are, do you need them? They answer can be 'yes', but know why. 


\section{Mitigation: Developers : libabigail}

Consider using abipkgdiff (from libabigail) to track your package ABI changes over time. The output is even more detailed with the debuginfo installed!

\$ abidiff 5.1.2-alpha/usr/lib64/liblzma.so.5 5.2.2/usr/lib64/liblzma.so.5

Functions changes summary: 0 Removed, 0 Changed, 0 Added function

Variables changes summary: 0 Removed, 0 Changed, 0 Added variable

Function symbols changes summary: 0 Removed, 3 Added function symbols not referenced by debug info Variable symbols changes summary: 0 Removed, 0 Added variable symbol not referenced by debug info

3 Added function symbols not referenced by debug info:

[A] 1zma_block_uncomp_encode@exz_5.2.2

[A] 1zma_cputhreads@@X_5.2.2

[A] 1zma_get_progress@@X_5.2.2

Additionally, libabigail provides the abicompat command for further review of a library's dependencies. The reports can be super helpful for determining compatibility and tracking changes over time. 


\section{Mitigation: Developers : symbols you choose}

How do you tell if a feature is "new"?

Look at the documentation for your library

- Is the feature you're using listed as coming with a newer version of the library than you've got installed? If so, this feature was backported to the EL source. When though?

- Was the library rebased "recently" in Stream? What does "recently" mean here?

\section{Easy way : test your binary with RHEL UBI}

The RHEL Universal Base Image (ebook link) is real RHEL. UBI is free to download and redistribute. "No subscription, login, or even registration is required for the UBI images."

If you picked a set of software behaviors for your application, know why you picked them. 


\section{Mitigation: Developers : RPM symbols}

Let RPM decide your binary dependencies.

Look at our xz rpms again, rpm -qp --requires xz-5.2.2-1.el7.x86_64.rpm Igrep liblzma

liblzma.so.5()(64bit)

liblzma.so.5(XZ_5.0)(64bit)

liblzma.so.5(XZ_5.1.2alpha)(64bit)

liblzma.so.5(XZ_5.2.2)(64bit)

RPM knows what we need. Setting a specific version of a binary package to required for a binary RPM is counterproductive. If you recompile the Source RPM it should generate the dependencies it needs. If you hard code a specific version of a binary you need, you are reducing the functionality. Do you need liblzma-5.2.2 or the symbols it provides? 


\section{Mitigation: Developers "surprise symbols"}

Looking back at our xz binary and the pthread_sigmask symbol selection. The liblzma library didn't specifically choose the new or old symbol. It just used the pthread headers on the system.

How do you force a specific symbol when multiple versions exist?

In GCC you can use this:

__asm__(".symver pthread_sigmask,pthread_sigmask@GLIBC_2.2.5");

to select a specific version rather than let the compiler/linker/library choose.

https://developers.redhat.com/blog/2019/08/01/how-the-gnu-c-library-handles-backward-compatibility/ 


\section{Mitigation: Developers "surprise symbols"}

The glibc library is probably the most likely to be a source of 'surprise symbols'. We can gather some useful stats from EL7 (in year 7 now) and security errata only maintenance. New features in EL7 at this point would be a surprise.

The /lib64/libc. so. 6 library from glibc-2.17-323.el7_9.x86_64 contains 2125 symbols which are scoped to multiple namespaces. Some of them are 'unscoped' versions of the 'scoped' version.

The original /lib64/libc.so. 6 published with EL7.0 (glibc-2.17-55.el7.x86_64) contains 2100.

Testing Methodology (better methodology: abidiff --no-default-suppression): readelf -s --wide ./lib64/libc.so.6 | awk '\{print $\$ 8\}$ ' | sort -u | cut -d '@' -f1 


\section{Mitigation: Developers things you "can't" do - extraction}

You "cannot" directly extract a specific symbol from a binary library. A binary library is a blob of instructions, not a file system directory of symbols or tar archive. This is something I know but often forget.

The binary has been optimized and the related jumps are relative to the location within the blob. If you disassemble it, it may be possible to reassemble a stub library with just a subset.

But if you've gotten this far down the chain a better question is, "Is this feature worth it?" or "Can I just ship the new library?" If you need a new feature, shipping the whole library makes a lot more sense since upstream probably tested it that way.

If you want to build a new binary that just contains the symbol you want, you can always edit the source and compile what you desire. And that is probably a lot less work than using a disassembler on an existing binary. 


\section{Mitigation: Developers - DANGEROUS THINGS}

ELF objects support an rpath value that you can use to tweak the symbol resolution. If you need this, read up on DT_RPATH vs DT_RUNPATH.

In RHEL, RPM will not let you package binaries with encoded rpath values! This is a good thing! Packages should either ship the libraries they need and put them in a rational place, or depend on the libraries already in a rational place.

If you are using rpath, you claim to know better than the system linker. Are you sure that is true? Spack does this because it knows where the libs go.

You can use patchelf to mess about with rpath settings on existing binaries.

Never ever do this on a file owned by a package manager.

Updates to the package will lose the change.

The installation is no longer repeatable by the package manager. 


\section{Mitigation: Operations}

What should operations do and think about? 


\section{Mitigation: Operations}

Use technologies that are linkage aware: ie package managers - not make install.

RPM uses objdump to evaluate what you need. It is tracking at "namespace/version" level, not each individual binary symbol. A badly behaving library may add symbols to an existing namespace. An older/existing copy might have that namespace, but not the new symbols. Adding a feature and not changing the version is a bad practice. File bugs on this if it happens!

The fastest way to diagnose this is probably via ldd (see note on safety in manpage) $\$$ ldd $-r$ xz \# el7 binary on el8

\section{[snip]}

undefined symbol: lzma_get_progress, version XZ_5.2.2

undefined symbol: lzma_stream_encoder_mt, version XZ_5.1.2alpha

undefined symbol: lzma_stream_encoder_mt_memusage, version XZ_5.1.2alpha

undefined symbol: lzma_cputhreads, version XZ_5.2.2 


\section{Mitigation: Operations workarounds}

If you find yourself needing these workarounds, you've hit a bug that should be filed with the package owner or person who gave you the binaries.

For applications run as non-root but a "non-daemon" user, the $\sim /$. local/lib directory is a place users can drop their own libraries and add to LD_LIBRARY_PATH. See also /.1ocal/bin and the XDG Base Directory Spec documentation.

For daemons running out of systemd, you can add another directory to the service's defined LD_LIBRARY_PATH as a drop-in.

If the application doesn't fit under these or within a container, l'd consider generating a flatpak to ensure any extra libraries are walled off from the rest of the system and that it can be trivially moved to a new box down the line. A "one time install" is never one time in my experience.

It is tempting to drop a config into /etc/ld. so. conf. $d$, but this pollutes the global linker space. It is tempting to use rpath to just fix things, but this is invisible and will get forgotten. 


\section{Concluding Thoughts}

What have we learned and how can we apply it? 


\section{Areas of Improvement}

Existing self tests are pretty good, but with your help they could be great! See $\%$ check in your favorite package! Or ask about next steps on centos-devel. Functional testing workloads are also welcome! This is how we help assure the quality of the released packages.

A Koji plugin to run abidiff against the artifacts from the last build would produce an amazing record of changes!

Different output formats for libabigail would be wonderful! Annotated sources? JSON? Something else? Make this data more digestible and more usable! 


\section{So, Will my CentOS Stream application run on RHEL?}

In practice the answer to this is a function of the RHEL ABI/API guarantee that we've been talking about and the mindset of the code developer.

Something built on CentOS Stream should run on RHEL if it was built with compatibility in mind.

If a binary has special requirements, there are a number of ways to work around the linker until the symbols are considered part of the consistency guarantee.

So, will my CentOS Stream application run on RHEL? I believe the answer is clearly "yes, this shouldn't be a problem". 


\section{Notice of Production}

Work supported by the Fermi National Accelerator Laboratory, managed and operated by Fermi Research Alliance, LLC under Contract No. DE-AC02-07CH11359 with the U.S. Department of Energy. The U.S. Government retains and the publisher, by accepting the article for publication, acknowledges that the U.S. Government retains a non-exclusive, paid-up, irrevocable, world-wide license to publish or reproduce the published form of this manuscript, or allow others to do so, for U.S. Government purposes. 


\section{Questions?}




\section{\#Fermilab}

